\title{
Effects of antiepileptic drugs on the serum folate and vitamin B12 in various epileptic patients
}

\author{
HONG-LI HUANG, HAO ZHOU, NUAN WANG and CHUN-YU YU \\ Department of Neurology, The First People's Hospital of Xuzhou, Xuzhou, Jiangsu 221000, P.R. China
}

Received January 8, 2016; Accepted August 1, 2016

DOI: 10.3892/br.2016.737

\begin{abstract}
Epilepsy is a common neurodegenerative disease with an increasing morbidity. Clinical treatment of epilepsy includes symptomatic treatment, etiological treatment, surgery and prevention. The aim of the present study was to determine the effects of antiepileptic drugs (AEDs) on serum folate and vitamin B12 in various epileptic patients, and to examine the correlation between these effects and secondary cerebrovascular events. A total of 68 epileptic patients, diagnosed between May 2012 and May 2014, were included in the present study. The study included 8 cases of autonomic seizures, 10 cases of absence seizures, 13 cases of complex partial seizures, 28 cases of generalized tonic-clonic seizures, and 9 cases of simple partial seizures. The patients received appropriate AED treatment according to the characteristics of epileptic seizure and the treatment guidance. The differences in the serum levels of folate and vitamin B12 in these patients, and the differences in the secondary cerebrovascular events in these patients after 1 year follow-up were analyzed. The difference in the AEDs used by various epileptic patients was statistically significant $(\mathrm{P}<0.05)$. The proportion of AED monotherapy in the autonomic seizure group and petit mal group was highest, and the proportion of two AED in combination with the psychomotor seizure, grand mal and simple partial seizure groups was highest. The serum levels of folate and vitamin B12 in these patients following treatment were significantly lower than those prior to treatment $(\mathrm{P}<0.05)$. The differences in the serum levels of folate and vitamin B12 in these groups following treatment were not statistically significant $(\mathrm{P}>0.05)$. The difference in the incidence of cerebrovascular events in these groups at follow up was not statistically significant $(\mathrm{P}>0.05)$. The multifactorial logistic regression analysis revealed that the serum levels of
\end{abstract}

Correspondence to: Dr Hao Zhou, Department of Neurology, The First People's Hospital of Xuzhou, 19 Zhongshanbei Road, Xuzhou, Jiangsu 221000, P.R. China

E-mail: hao_zhou9@163.com

Key words: antiepileptic drugs, folate, vitamin B12, cerebrovascular events, independent risk factors folate and vitamin B12 were the independent risk factors for epilepsy with secondary cerebrovascular events [folate: odds ratio $(\mathrm{OR})=0.536, \mathrm{P}=0.039$; vitamin: $\mathrm{OR}=0.382, \mathrm{P}=0.041]$. In conclusion, various AEDs may decrease the serum levels of folate and vitamin B12 and affect the secondary cerebrovascular events in various epileptic patients. Thus, regular supplementation of folate and vitamin B12 may be an option.

\section{Introduction}

Epilepsy is a syndrome caused by transient cerebral dysfunction due to human brain electrical activity disorder and is a common nervous system disease with a worldwide prevalence of $7.0 \%$ (1). Additionally, epilepsy has an increasing annual morbidity, second only to stroke (2). The clinical treatment of epilepsy includes symptomatic treatment, etiological treatment, surgery, and the prevention of the cause (3). Mounting evidence (4) suggests that long-term administration of antiepileptic drugs (AEDs) may affect the metabolism of serum folate and vitamin B12, further increasing the serum level of homocysteine and leading to cerebrovascular events.

However, the abovementioned studies have not described the effect of AED on epilepsy in detail, and whether epilepsy with secondary cerebrovascular events was associated with the metabolic disorder of serum folate and vitamin B12 (5). The present study aimed to determine the effects of AEDs on the serum levels of folate and vitamin B12 in various epileptic patients, and examined the correlation between these effects and secondary cerebrovascular events.

\section{Subjects and methods}

General information. A total of 68 patients, diagnosed with epilepsy at the The First People's Hospital of Xuzhou (Jiangsu, China) between May 2012 and May 2014, were successively selected in the treatment group. All 68 patients were diagnosed with epilepsy based on the clinical symptoms and active electroencephalogram. The exclusion criteria were: i) age $<18$ years; ii) pregnancy; iii) primary cerebral vascular disease, high agglutination, severe heart, liver and kidney dysfunction, malignancy; iv) persistent and uncontrollable epileptic seizure; v) no history of regular administration of folate and vitamin B12; and vi) refusal to participate in the study. Of the 68 patients, there were 8 cases of autonomic seizures, 10 cases of absence seizures (petit mal), 13 cases of complex partial 
Table I. The AEDs used by various epileptic patients [no., (\%)].

\begin{tabular}{lcccr}
\hline Group & No. of patients & Monotherapy & 2 AEDs in combination & $\geq 3$ AEDs in combination \\
\hline Autonomic seizures & 8 & $5(62.5)$ & $2(25.0)$ & $1(12.5)$ \\
Petit mal & 10 & $6(60.0)$ & $2(20.0)$ & $2(20.0)$ \\
Psychomotor seizures & 13 & $4(30.8)$ & $6(46.2)$ & $3(23.1)$ \\
Grand mal & 28 & $5(17.9)$ & $13(46.4)$ & $10(35.7)$ \\
Simple partial seizures & 9 & $3(33.3)$ & $4(44.4)$ & $2(22.2)$ \\
$\chi^{2}$ test & & 2.635 & 2.856 & 2.745 \\
P-value & & 0.034 & 0.035 & 0.032 \\
\hline
\end{tabular}

AED, antiepileptic drug.

seizures (psychomotor seizures), 28 cases of generalized tonic clonic seizures (grand mal), and 9 cases of single partial seizures. The autonomic seizure group included 4 males and 4 females, with a mean age of $46.3 \pm 5.4$ years (23-58 years) and a mean disease course of $3.4 \pm 1.2$ years ( 1 month- 6 years). The petit mal group included 6 males and 4 females, with a mean age of $46.7 \pm 6.3$ years (25-65 years) and a mean disease course of $3.6 \pm 1.1$ years (1.5 months- 8.5 years). The psychomotor seizure group included 7 males and 7 females, with a mean age of 45.9 \pm 3.4 years (32-69 years) and a mean disease course of $3.5 \pm 1.4$ years (2.3 months-7.5 years). The grand mal group included 15 males and 13 females, with a mean age of $46.6 \pm 3.5$ years (21-66 years), and a mean disease course of $3.7 \pm 1.4$ years (1.3 months- 8.9 years). The single partial seizure group included 4 males and 5 females, with a mean age of $47.4 \pm 5.2$ years (31-74 years), and a mean disease course of $3.5 \pm 1.2$ years (2.2 months-9.6 years). These patients were comparable with respect to gender, age, and disease course $(\mathrm{P}>0.05)$. Approval for the study was obtained from the local Ethics Committee. Informed consent was obtained from the patients and family members.

Methods. The patients received appropriate AED treatment according to the characteristics of epileptic seizure and the treatment guidance. The differences in the serum levels of folate and vitamin B12 in these patients, and the differences in the secondary cerebrovascular events in these patients after the 1 year follow-up were analyzed. The used AEDs included carbamazepine $(0.2 \mathrm{~g}$, bid po), phenytoin $(0.1 \mathrm{~g}$, tid po), valproate (0.3 g, tid po), and lamotrigine (0.05 g, qd po). These drugs were administered as monotherapy, or 2 AEDs or 3 AEDs in combination to control the symptoms and prevent recurrence. Blood $(5 \mathrm{ml})$ was collected from the ulnar vein of fasting and resting patients, $2 \mathrm{ml}$ venous blood was agitated and the plasma was isolated. The remaining $3 \mathrm{ml}$ blood was used to isolate the serum after coagulation. The ACS:180SE automatic chemiluminescence detector (Bayer, Pittsburgh, PA, USA) and the reagent kit were used to detect the serum levels of folate and vitamin B12, following the manufacturer's instructions. The definition of secondary cerebrovascular events was those events caused by hypertension, diabetes, coronary heart disease, and hyperlipidemia. Cerebrovascular events prior to epilepsy were excluded. Cerebrovascular events included ischemic and hemorrhagic stroke.
Statistical analysis. SPSS 17.0 software (IBM SPSS, Armonk, NY, USA) was used for the statistical analysis. Quantitative data were presented as mean \pm standard deviation, and the intergroup comparison was performed using a t-test. Qualitative data were presented as a number or $\%$, and the intergroup comparison was performed using the Chi-square test. The correlation between the serum levels of folate and vitamin B12 and the serum level of homocysteine was analyzed using the Pearson test. The screening of the risk factors for epilepsy with secondary thrombosis was analyzed using logistic regression analysis with the stepwise backward method. $\mathrm{P}<0.05$ was considered to indicate a statistically significant difference.

\section{Results}

The AEDs used by various epileptic patients. As shown in Table I, the difference in the AEDs used by various epileptic patients was statistically significant $(\mathrm{P}<0.05)$. The proportion of AED monotherapy in the autonomic seizure $(62.5 \%)$ and petit mal groups $(60.0 \%)$ was highest, and the proportion of 2 AEDs in combination, in the psychomotor seizure $(46.2 \%)$, grand mal (46.4\%) and simple partial seizure groups (44.4\%), was highest.

Serum levels of folate and vitamin B12 in various epileptic patients. As shown in Table II, the differences in the serum levels of folate and vitamin B12 in these patients prior to treatment were not statistically significant $(\mathrm{P}>0.05)$. The serum levels of folate and vitamin $\mathrm{B} 12$ following treatment were significantly lower than those prior to treatment $(\mathrm{P}<0.05)$. The differences in the serum levels of folate and vitamin B12 in these groups following treatment were not statistically significant $(\mathrm{P}>0.05)$.

Incidence of secondary cerebrovascular events in various epileptic patients. The difference in the incidence of secondary cerebrovascular events in various epileptic patients at follow-up was not statistically significant $(\mathrm{P}>0.05)$. There was 1 case $(12.5 \%)$ in the autonomic seizure group, 1 case $(10.0 \%)$ in the petit mal group, 1 case $(7.7 \%)$ in the psychomotor seizure group, 2 cases $(7.1 \%)$ in the grand mal group, and 1 case $(11.1 \%)$ in the simple partial seizure group $\left(\chi^{2}=0.523, \mathrm{P}=0.637\right)$. In the multifactorial logistic regression analysis, epilepsy with secondary cerebrovascular events was identified as the dependent variable, and the patient's gender, age, disease course, epilepsy type, AEDs, homocysteine, folate, and vitamin B12 were the 
Table II. Serum levels of folate and vitamin B12 in various epileptic patients.

\begin{tabular}{|c|c|c|c|c|}
\hline \multirow[b]{2}{*}{ Group } & \multicolumn{2}{|c|}{ Folate $(\mathrm{Q} / \mathrm{pg} \cdot \mathrm{l})$} & \multicolumn{2}{|c|}{ Vitamin B12 ( $/ \mathrm{pgg} \cdot \mathrm{l})$} \\
\hline & Before treatment & After treatment & Before treatment & After treatment \\
\hline Autonomic seizures & $11.5 \pm 2.7$ & $6.5 \pm 1.2^{\mathrm{a}}$ & $492.3 \pm 50.1$ & $352.7 \pm 34.2$ \\
\hline Petit mal & $12.2 \pm 2.6$ & $5.9 \pm 1.3^{\mathrm{a}}$ & $505.2 \pm 45.3$ & $367.5 \pm 42.1$ \\
\hline Psychomotor seizures & $11.6 \pm 2.1$ & $6.1 \pm 1.4^{\mathrm{a}}$ & $521.3 \pm 42.5$ & $378.9 \pm 35.6$ \\
\hline Grand mal & $12.3 \pm 1.9$ & $6.4 \pm 1.2^{\mathrm{a}}$ & $495.6 \pm 33.4$ & $349.2 \pm 36.4$ \\
\hline Simple partial seizures & $11.9 \pm 2.2$ & $6.3 \pm 0.9^{\mathrm{a}}$ & $492.8 \pm 34.1$ & $354.8 \pm 41.2$ \\
\hline$\chi^{2}$ test & 0.638 & 0.526 & 0.527 & 1.659 \\
\hline P-value & 0.412 & 0.624 & 0.935 & 0.821 \\
\hline
\end{tabular}

${ }^{\mathrm{a} C o m p a r e d ~ w i t h ~ b e f o r e ~ t r e a t m e n t ~}(\mathrm{P}<0.05)$.

independent variables. It was found that the serum levels of folate and vitamin B12 were the independent risk factors for epilepsy with secondary cerebrovascular events [folate: odds ratio $(\mathrm{OR})=0.536, \mathrm{P}=0.039$; vitamin $\mathrm{B} 12$ : $\mathrm{OR}=0.382, \mathrm{P}=0.041]$.

\section{Discussion}

Epilepsy is a common neurodegenerative disease with an increasing morbidity. The etiology of epilepsy includes: i) Intracranial tumor, such as glioma, meningioma, and astrocytoma, which is prevalent in the elderly, where the main manifestation includes complex partial seizures and generalized tonic-clonic seizures (6); ii) cerebrovascular diseases and cerebral vascular malformation in the young and elderly, where the manifestation can be any type of seizure (7); and iii) nutritional and metabolic disorders, such as hypoglycemia, hyperthyroidism and diabetes. Vitamin B6 deficiency can also cause epileptic seizure. The main manifestation is autonomic seizures and simple partial seizures. iv) Degenerative diseases can increase the incidence of epilepsy, such as tuberous sclerosis, and Alzheimer's disease, with the main manifestation being complex partial seizures (8). v) High fever and convulsion can cause cerebral ischemia, hypoxia, and edema, and form epileptic foci. This is the main etiology of neonatal febrile seizures. The main manifestation is absence seizures. vi) Brain trauma can cause softening of brain tissue to form epileptic foci. This is the main etiology of neonatal epileptic seizure. The main manifestation is absence seizures and complex partial seizures; and viii) infection.

Clinical treatment of epilepsy primarily involves medication therapy. It has been demonstrated that although long-term administration of AEDs relieve symptoms, the levels of folate and vitamin B12 may be altered and associated with the type and dose of AEDs, epilepsy type, disease course, and individual gene (6). However, little evidence is available regarding this observation. The current clinical studies focus on single sites, have unfocused observation, and are inconsistent or have contradictory conclusions. Thus, administering clinical treatment was difficult (9-11).

The present study determined the effects of AEDs on the serum levels of folate and vitamin B12 in various epileptic patients, and examined the correlation between these effects and secondary cerebrovascular events. The difference in the AEDs administered to various epileptic patients was statistically significant. The serum levels of folate and vitamin B12 were decreased following treatment, and the differences were statistically significant. By contrast, the differences in the serum levels of folate and vitamin B12 between the groups after treatment were not statistically significant. The difference in the incidence of secondary cerebrovascular events between the groups at follow-up was not statistically significant. The multifactorial logistic regression analysis revealed that the serum levels of folate and vitamin B12 may be independent risk factors for epilepsy with secondary cerebrovascular events (folate: $\mathrm{OR}=0.536, \mathrm{P}=0.039$; vitamin: $\mathrm{OR}=0.382, \mathrm{P}=0.041)$. Inconsistent with previous studies $(8,9)$, the present findings demonstrated that AEDs may decrease the levels of folate and vitamin B12 in the body. However, this effect was irrelevant to the type of epilepsy. The secondary cerebrovascular events after epilepsy were also associated with the levels of folate and vitamin B12. AEDs, such as carbamazepine and phenytoin sodium, may markedly alter the level of folate in epileptic patients. The mechanism included $(12,13)$ : i) Increased $\mathrm{pH}$ in the gastrointestinal tract and decreased activities in the intestinal enzymes impaired the conversion from polyglutamate folate into folate, leading to lower absorption of folate; ii) phenytoin sodium was similar to folate in the structure; thus, there was competition between the two drugs, leading to decreased folate binding to the folate receptors on intestinal mucosal cells and decreased absorption of folate; iii) phenytoin sodium may decrease the activity of folate-metabolizing enzymes, such as methylene synthase; as a result, the synthesis of folate was impaired; and iv) phenytoin sodium and carbamazepine increased the activity of hepatic microsomal enzymes, accelerated the metabolism of folate, and decreased the level of folate in body.

Folate is a nutrient in many biochemical reactions in the body, and an important factor for human health. As an important component in the synthesis of protein and nucleic acid, folate is predominantly involved in the metabolism of one carbon unit. In the case of folate deficiency, the frequency of chromosome breakage or translocations is higher, leading to an abnormal karyotype. Vitamin B12 occurs mainly in the form of glutamyl and methyl glutamyl coenzymes. This vitamin 
is linked to cobalt ion, and is known as cyanocobalamin ammonia, which is a green crystal. Vitamin B12 has two functions (14-16): i) It acts as the co-factor of methyl transferase, where it is involved in the synthesis of amino acids and nuclei acid. When the methyl group in methyltetrahydrofolic acid is transferred to the receptor of methyl, such as homocysteine, tetrahydrofolic acid is produced, leading to reduced synthesis of nucleic acid and protein; and ii) to promote the transport and storage of folate. It has been reported (7) that in the case of folate deficiency, transport of the methyl group from homocysteine to methionine was impaired, leading to decreased intracellular level of folate and folate storage in hepatocytes.

In summary, various AEDs decreased the serum levels of folate and vitamin B12 in various epileptic patients to a certain extent, and affect the secondary cerebrovascular events. Thus, regular administration of folate and vitamin B12 may be an option. However, the mechanism underlying the effects of folate and vitamin B12 on the secondary cerebrovascular events remains to be identified. Therefore, additional studies should be conducted to confirm the results.

\section{References}

1. Alvim MK, Coan AC, Campos BM, Yasuda CL, Oliveira MC, Morita ME and Cendes F: Progression of gray matter atrophy in seizure-free patients with temporal lobe epilepsy. Epilepsia: Feb 11, 2016 (Epub ahead of print).

2. Zhu F, Lang SY, Wang XQ, Shi XB, Ma YF, Zhang X, Chen YN, and Zhang JT: Long-term effectiveness of antiepileptic drug monotherapy in partial epileptic patients: A 7-year study in an epilepsy center in China. Chin Med J (Engl) 128: 3015-3022, 2015.

3. Moura LM, Mendez DY, De Jesus J, Andrade RA and Hoch DB: Quality care in epilepsy: Women's counseling and its association with folic acid prescription or recommendation. Epilepsy Behav 44: 151-154, 2015.

4. Linnebank M, Moskau S, Semmler A, Widman G, Stoffel-Wagner B, Weller M and Elger CE: Antiepileptic drugs interact with folate and vitamin B12 serum levels. Ann Neurol 69: 352-359, 2011.
5. Belcastro V and Striano P: Vitamin B12, folate and hyperhomocysteinemia in patients with epilepsy. Ann Neurol 69: 1067-1068, author reply 1068, 2011.

6. Hao N, Xia W, Tang Y, Wu M, Jiang H, Lin X, Liu J and Zhou D: Periconceptional folic acid supplementation among pregnant women with epilepsy in a developing country: A retroprospective survey in China. Epilepsy Behav 44: 27-34, 2015.

7. Bochyńska A, Lipczyńska-Łojkowska W, Gugała-Iwaniuk M, Lechowicz W, Restel M, Graban A, Lipska B and Ryglewicz D: The effect of vitamin B supplementation on homocysteine metabolism and clinical state of patients with chronic epilepsy treated with carbamazepine and valproic acid. Seizure 21: 276-281, 2012.

8. Oz O, Gökçil Z, Bek S, Cakir E and Odabaşi Z: Is asymmetric dimethylarginine responsible for the vascular events in patients under antiepileptic drug treatment? Epilepsy Res 87: 54-58, 2009.

9. Belcastro V and Striano P: Antiepileptic drugs, hyperhomocysteinemia and B-vitamins supplementation in patients with epilepsy. Epilepsy Res 102: 1-7, 2012.

10. Kosyfaki P, Woerner W and Att W: Prosthodontic treatment in a partially edentulous patient with a complex medical history of epilepsy and deep vein thrombosis: A case report. Quintessence Int 42: 365-373, 2011.

11. Grover PJ, Jayaram R and Madder H: Management of cerebral venous thrombosis in a patient with Lane-Hamilton syndrome and coeliac disease, epilepsy and cerebral calcification syndrome. Br J Neurosurg 24: 684-685, 2010.

12. Brazzelli V, Grasso V, Fornara L, Moggio E, Gamba G, Villani S and Borroni G: Homocysteine, vitamin B12 and folic acid levels in psoriatic patients and correlation with disease severity. Int $\mathbf{J}$ Immunopathol Pharmacol 23: 911-916, 2010.

13. Salemi G, Gueli MC, D'Amelio M, Saia V, Mangiapane P, Aridon P, Ragonese P and Lupo I: Blood levels of homocysteine, cysteine, glutathione, folic acid, and vitamin B12 in the acute phase of atherothrombotic stroke. Neurol Sci 30: 361-364, 2009.

14. Cakmak SK, Gül U, Kiliç C, Gönül M, Soylu S and Kiliç A: Homocysteine, vitamin B12 and folic acid levels in psoriasis patients. J Eur Acad Dermatol Venereol 23: 300-303, 2009.

15. Zhou K, Zhao R, Geng Z, Jiang L, Cao Y, Xu D, Liu Y, Huang L and Zhou J: Association between B-group vitamins and venous thrombosis: Systematic review and meta-analysis of epidemiological studies. J Thromb Thrombolysis 34: 459-467, 2012.

16. Remacha AF, Souto JC, Piñana JL, Sardà MP, Queraltó JM, Martí-Fabregas J, García-Moll X, Férnandez C, Rodriguez A and Cuesta J: Vitamin B12 deficiency, hyperhomocysteinemia and thrombosis: A case and control study. Int J Hematol 93: 458-464, 2011. 